\title{
Society of Hematologic Oncology (SOHO) State of the Art Updates and Next Questions: Myelodysplastic Syndromes
}

\author{
Valeria Santini
}

In the past few months, 2 main streams of research have dominated the panorama of myelodysplastic syndrome (MDS) investigations: deepening the insight into the pathogenic role, hierarchy, and prognostic effect of somatic mutations and, as a consequence, into the effect of inherited congenital predisposing conditions and the second, quite interlinked with the first, analyzing inflammation and innate immunity in patients with MDS. The research devoted to clarifying the mechanisms of action and mechanisms of resistance to hypomethylating agents has also advanced, mostly resulting from different approaches to the study of DNA methylation. Recent observations have reinforced support for targeted therapies for selected subgroups of MDS patients.

Clinical Lymphoma, Myeloma \& Leukemia, Vol. 18, No. 8, 495-500 ๔ 2018 Elsevier Inc. All rights reserved.

Keywords: Inflammation, MDS, Methylation, Somatic mutations, Targeted therapy

\section{Introduction}

The diagnosis of myelodysplastic syndromes (MDSs) has been based on the morphologic assessment of dysplastic hematopoietic progenitor cells of the various lineages, with the characteristic features of dysplasia, which are subtly different from the dysplasia secondary to inflammation or chronic disease, and a predetermined minimum percentage $(10 \%)$ of abnormal cells. ${ }^{1}$ However, this diagnostic approach presents relevant difficulties and therefore requires high expertise and is time consuming. The analysis of MDS bone marrow aspirates is subjective, depending on the interpretation of the morphologic features by the physician performing the analysis. ${ }^{2}$ These could be the reasons why some diagnoses of MDS are dubious and require confirmation by abnormal cytogenetic and flow cytometric analysis findings. ${ }^{2,3}$ The latter method has been increasingly used in the idea of facilitating detection of marrow dysplasia. However, high expertise is also required for the evaluation, which has a number of limitations, diagnostically determinant. ${ }^{3}$ Although in the scientific MDS community, all these issues have been frequent subjects of discussion, it is clear that the presence of genetic abnormalities is fundamental to the diagnosis and prognosis of MDS. 4,5

MDS Unit, Azienda Ospedaliero Universitaria Careggi, University of Florence, Florence, Italy

Submitted: May 11, 2018; Accepted: May 17, 2018; Epub: May 23, 2018

Address for correspondence: Valeria Santini, MD, MDS Unit, Azienda Ospedaliero Universitaria Careggi, University of Florence, Largo Brambilla 3, Florence 50134, Italy E-mail contact: santini@unifi.it
The international prognostic scoring system (IPSS) and IPSSrevised (IPSS-R) ${ }^{5,6}$ are routinely applied to stratify MDS, from a prognostic viewpoint, into lower risk and higher risk. IPSS-R prognostic scoring is strongly influenced by the classified cytogenetic abnormalities and, less than with the previous IPSS version, the marrow blast percentage and depth of cytopenia. The risk is defined by these variables and is related to overall survival and the propensity to develop acute leukemia and, in fact, allow for good stratification of MDS patients. In the IPSS-R, the presence of dysplasia and the number of dysplastic lineages were not given relevant weight, although both are determinant for the World Health Organization (WHO) diagnosis of MDS. Nevertheless, the IPSS-R is good but not sufficient to encompass the level of recognized heterogeneity of MDS in terms of outcome and disease behavior. The WHO-based prognostic scoring system-revised, another prognostic scoring system, also relies on the morphologic diagnosis of the type of dysplasia and seems to be more successful in the prognostic stratification of MDS cases.

In the past few years, the increasingly sophisticated methods of molecular analysis have allowed for the characterization of the frequent somatic mutations present in MDS. Somatic mutations are present in nearly $90 \%$ of cases of MDS (Figure 1). Because of their high frequency, knowledge of the specific mutations could improve the prognostic evaluation of the $50 \%$ to $60 \%$ of MDS patients with a normal karyotype. ${ }^{8}$ However, the effect of the 40 more frequent somatic mutations on the natural history of MDS has not yet been completely ascertained. ' The recent WHO classification has included the spliceosome gene mutation SF3B1 as the only 


\section{SOHO Updates and Open Questions for MDS}

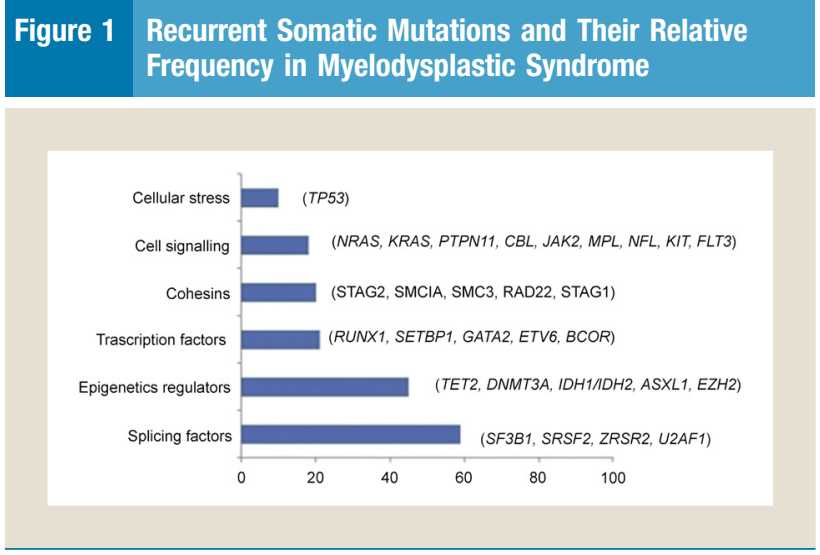

determinant for the MDS subtype. ${ }^{1}$ The importance of single mutations, co-mutations, and variant allele frequency of each mutation is under study by several groups. The huge international effort led by the International Working Group for the Prognosis of MDS is evaluating the clinical significance of somatic mutations using next generation sequencing (NGS) of material from $\sim 5000$ cases of MDS with complete clinical annotations obtained from several hematologic centers worldwide. The results will give relevant information to the community and will most probably drive future classification of these diseases. Although the final results of that study are not yet available, the investigators have demonstrated the relevance of the number and types of mutations in MDS in a smaller cohort of cases. ${ }^{9-12}$

With these observations, it is intuitive that the diagnosis of MDS is shifting from the use of morphology to molecular analysis. In the past few months, even the role of cytogenetic evaluation has been under discussion. Moreover, it is without doubt true that cytopenias without dysplasia can be difficult, and the definite diagnosis of their nature is often a challenge. The question remains regarding how to diagnosis MDS and the definition of MDS, and which will be the most reliable parameters and methods to apply. However, the "molecular approach" to hematopoiesis, which seemed able to solve the difficulties related to the diagnosis of MDS and its heterogeneity has provided information on a very complex scenario and rendered diagnostic applications in the context of MDS less straightforward.

\section{Clonal Hematopoiesis and the Difficult Diagnosis of MDS}

The existence of clonal hemopoiesis, which does not always correlate with neoplastic diseases, has long been known and assessed using different methods. ${ }^{13}$ These include evaluating the phenotype with flow cytometry and protein isoform determination, evaluating the genotype with $\mathrm{X}$ gene inactivation, T-cell receptor rearrangements and immunoglobulin rearrangements using cytogenetics/ array comparative genomic hybridization/single nucleotide polymorphism array, and, more recently, NGS.

Deep sequencing techniques have demonstrated the incidence and frequency of clonal hematopoiesis. In 2014, 3 relevant studies $^{14-16}$ reported the presence of the somatic mutations most frequently observed in MDS in very large non-MDS populations. Mutations of DNMT3a, ASXL1, TET2, Jak2, TP53, and SF3B1 genes and others were reported to be age related and to correlate with adverse outcome and the propensity to develop coronary heart disease and hematologic neoplasms. ${ }^{14-16}$ Subsequent to these observations, some small acronyms were created to define the different conditions and were added to the already used ICUS (idiopathic cytopenia of unknown significance) and IDUS (idiopathic dysplasia of unknown significance). These included CHIP/ARCH (clonal hemopoiesis of indeterminate potential/age-related clonal hemopoiesis) and CCUS (clonal cytopenia of unknown significance; Figure 2). ${ }^{17}$ For CHIP, clonality has been defined by the presence of MDS-associated genes, including DNMT3A, ASXL1, TET2, and JAK2, with loss of function. Individuals with CHIP have no clinical disorder and little propensity to develop MDS (0.5\%-1\% annually). However, CHIP is present in the same population that usually develops MDS (ie, $15 \%$ of those aged $>70$ years); thus, the parallel term ARCH was created. It is not clear whether this event is triggered by specific factors such as smoking, radiation, chemotherapy, or inflammation, is a stochastic event, or occurs with hereditary or predisposing conditions. ${ }^{17}$ However, CHIP, which has somehow been assimilated to MGUS (monoclonal gammopathy of undetermined significance) and MBL (monoclonal B-cell lymphocytosis), is not so innocent. It can be a precursor to therapy-related MDS and therapy-related acute myeloid leukemia (AML) in cancer patients after chemotherapy. ${ }^{19}$ CHIP has been demonstrated in $62 \%$ of cases before therapy-related AML/MDS compared with CHIP in $27 \%$ of controls. The variant allele frequency (VAF) generally increases in the case of progression to MDS or AML (Figure 2). Moreover, exposure to radiation therapy or tobacco use increases the risk of CHIP/ARCH more than chemotherapy exposure and has been found in $\sim 25 \%$ of solid tumor patients. ${ }^{20}$ Thus, the presence of CHIP/ARCH should be carefully evaluated before therapeutic decisions. In addition, it could be more common than that reported in hotspot-focused studies. ${ }^{20}$

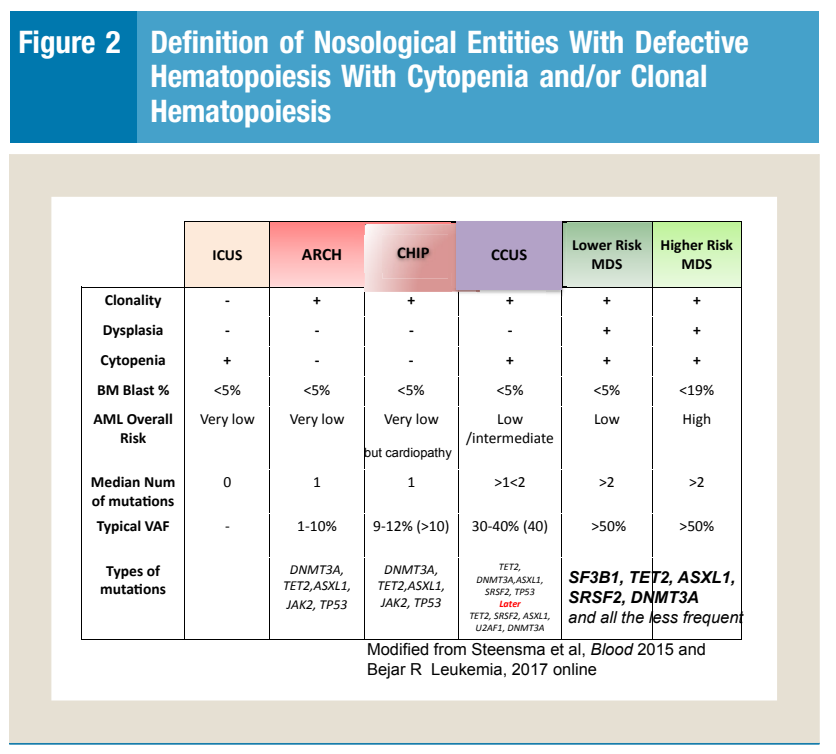

Abbreviations: $\mathrm{BM}=$ bone marrow; $\mathrm{BSC}=$ best supportive care; CCUS $=$ clonal cytopenias of undetermined significance; GF = hematopoietic growth factor (eg, epoetin); HMA = hypomethylating agent (eg, azacitidine); HSCT = hematopoietic stem cell transplantation; IMiD = immunomodulatory drug (eg, lenalidomide); IST = immunosuppressive therapy; Obs $=$ observation. Modified, with permission, from Steensma et $\mathrm{al}^{17}$ and Bejar et al. ${ }^{18}$ 
Therefore, it is necessary to determine the support that deep sequencing techniques might provide to the diagnosis of MDS in difficult cases. The presence of somatic mutations in patients with cytopenia (ie, CCUS) is predictive of the evolution (14 times greater probability) to MDS compared with that of unmutated ICUS. ${ }^{21}$ CCUS is a clonal condition, without evidence of relevant clinical alterations; however, an increase in clonal burden leads to progression to defined overt $\mathrm{MDS}^{18}$ (Figure 2). In contrast, the number of somatic mutations $(>2)$ and the size of the mutant clone $(\mathrm{VAF}>0.10)$ were shown to have significant predictive value for the diagnosis of MDS. ${ }^{21}$ However, not all mutations have the same value, and specific MDS mutations such as spliceosome gene mutations will be more strongly suggestive of a myeloid neoplasm. ${ }^{21}$ Therefore, careful integration of the molecular data could support the diagnosis in the negative or positive sense for those patients with cytopenias without karyotype abnormalities and scarce marrow dysplasia. However, prospective studies are needed. Although routine NGS could be helpful in defining the presence and size of the mutations in suspected MDS cases, a number of open questions remain before implementing this approach into routine clinical practice. In summary, in 2018, we can affirm that mutations cannot be used as surrogate for the clinical diagnosis of MDS (with exclusion of SF3B1 in the presence of 5\% ring sideroblasts in accordance with the WHO guidelines). If somatic mutations are present, it is mandatory to evaluate the type and VAF, especially in elderly patients. CCUS patients require very careful monitoring. The presence of CHIP has clinical implications for patients with solid tumors regarding the choice of therapy. However, the absence of somatic mutations in patients with ICUS or IDUS might only suggest and not definitively exclude an MDS diagnosis.

\section{Somatic Mutations in MDS: Prognostic Value and Therapeutic Targeting}

If the "molecular diagnosis" of MDS still presents problems, the role of somatic mutations as prognostic indicators has become rather consolidated. ${ }^{10-12}$ First, the number of somatic mutations carried by a patient with MDS will correlate with leukemia-free survival (worst with $>6$ mutations). ${ }^{10}$ However, it has also been correlated with a significantly inferior response to erythropoietic-stimulating agents in lower risk MDS cases in which the clinical features predicted the response $^{22}$ to hypomethylating agents (HMAs) $)^{23}$ and lenalidomide in non-del5q MDS. ${ }^{24}$ The occurrence of multiple mutations might, therefore, be considered a hallmark of aggressive disease and resistance to standard treatments.

The independent prognostic value of specific mutations was ascertained years earlier for MDS presenting with TP53, EZH2, ETV6, RUNX1, and ASXL1, which are predictors of poor overall survival. $^{25}$ In a particular subtype of MDS, with del5q, a great proportion of patients will have no or only 1 somatic mutation. Only 7 genes have shown mutation frequencies $>5 \%$ : SF3B1, DNMT3A, TP53, TET2, CSNK1A1, ASXL1, JAK2. Also, their relative pattern of frequency has been similar to that of all MDS cases, with the exception of TP53, which has shown more frequent mutations. ${ }^{26}$ TP53 mutations in MDS with del5q confer a poor prognosis, with a decreased response to lenalidomide and a greater propensity to progression to AML in a patient with MDS with an otherwise good outcome. ${ }^{27}$ The acquisition of TP53 and expansion of the mutated clone seems to occur during lenalidomide treatment. $^{27}$ The evaluation of TP53 mutations and the size of the mutated clone should thus be evaluated in all cases of MDS with del5q found at baseline owing to the evident clinical implications.

In terms of prognostic importance, not all mutations are equal, and their effect depends on the presence of co-mutations and the phenotype of MDS in which they arise, allowing for a possible molecular classification of MDS. ${ }^{28}$ TP53, U2AF1, RUNX1, ASXL1, EZH2, and SF3B1 are significant for overall survival on multivariate analysis for all MDS subtypes. In particular, the SF3B1 mutation is a major clustering criterion per se and identifies a distinct subset of MDS with a favorable prognosis, irrespective of the classification criteria used and the presence of ring sideroblasts. $^{28,29}$ Thus, it has been included in the most recent WHO classification. $^{1}$

The pathogenesis of MDS is becoming clearer owing to the evaluation of genetic alterations (both mutations and copy number alterations) that modify the molecular pathways and link genotype to phenotype. The increasing knowledge of the events leading to these diseases has rendered their modulation at least thinkable and has already supported more focused therapeutic decisions. ${ }^{30}$ In practice, evaluation of the mutational pattern of MDS in patients undergoing hematopoietic stem cell transplantation (HSCT) might in principle provide important independent prognostication in addition to the clinical factors. Mutations of TP53 were reported by 3 different investigators as the most potent indicators of a dismal outcome after HSCT. ${ }^{31-33}$ If TP53 is unmutated, the presence of ASXL1 and RUNX1 ${ }^{31}$ or RAS pathway mutations in MDS/ myeloproliferative neoplasm and JAK2 mutations ${ }^{32}$ are predictors of a poor outcome.

Although the pathogenetic and prognostic role played by specific mutations and co-mutations has been investigated, another important aspect of research is the importance of the hierarchical clonal order of these somatic mutations in determining the MDS phenotype. Founder mutations compared with subclonal mutations and the dynamic characteristics of specific mutations (sweeping, persistent, or vanishing) all contribute to generate the heterogeneity of subtypes of MDS, their clonal evolution, and differing natural histories. ${ }^{34}$ The top 5 dominant mutations are, as expected, SF3B1, TET2, SRSF2, DNMT3A, and ASXL1. Somatic mutations in MDS constitute possible therapeutic targets, and several mutationspecific agents are under investigation in clinical studies.

Isocitrate dehydrogenase IDH1 and IDH2 genes are not very frequently mutated in MDS (5\%-12\% of cases). However, 2 specific inhibitors, ivosidenib (AG-120) and enasidenib (AG-221), act by reducing the abnormal production of 2-hydroxyglutarate and promote MDS cellular differentiation. However, these do not necessarily eliminate the IDH1 or IDH2 mutated clones or change their VAFs. These agents are under clinical evaluation for MDS. The preliminary results of a phase I clinical trial (ClinicalTrials.gov identifier, NCT02074839) with ivosidenib in IDH1-mutated R7R AML and MDS indicated a $21 \%$ complete remission and $41 \%$ overall response rate. ${ }^{35}$ Enasidenib has been approved by the Food and Drug Administration for IDH2-mutated relapsed AML and was used for $30 \mathrm{AML} / \mathrm{MDS}$ patients in the registration trial with an overall response rate of $40 \%$. For the patients with refractory or 


\section{SOHO Updates and Open Questions for MDS}

relapsed MDS after HMAs and carrying mutated IDH2, 50\% showed a treatment response. ${ }^{36}$

Spliceosome gene mutations are very frequent in MDS, and targeting agents have been investigated. The pleiotropic function of many of these genes has led to first failure in therapeutic attempts with the E7107 compound owing to the important side effects. ${ }^{37}$ Clinical studies of the oral spliceosome inhibitor H3B-8800 for MDS carrying mutations in various spliceosome genes are ongoing after the preclinical demonstration of their activity because H3B8800 binds to the mutant and wild-type SF3b complex but exerts its cytotoxic activity only on mutant cells, exploiting the "spliceosome insufficiency" of the mutated cells. ${ }^{38}$ Although not directed specifically against SF3B1-mutated MDS, the 2 transforming growth factor- $\beta$ pathway inhibitors luspatercept and sotatercept have demonstrated in phase II studies selective clinical activity in this subtype of MDS. ${ }^{39,40}$ Therapy with these agents, especially luspatercept, has led to a $>70 \%$ response rate in SF3B1-mutated cases, with rather stable transfusion independence in those with lower risk severely anemic MDS. It is possible that such agents are acting only indirectly in cases in which transforming growth factor$\beta$ pathways is highly stimulated instead of on the mutation itself.

Given the important role of mutant TP53, it constitutes a relevant target for therapy. Although not yet studied in MDS, it has become clear that TP53 targeting is possible, both directly and indirectly, with a series of agents such as idasanutlin, ${ }^{41}$ an MDM2 inhibitor in evaluation to treat AML, and similar to APR246, ${ }^{42}$ which stabilizes the mutant TP53 in its wild-type conformation, activating downstream pathways and restoring tumor suppressor function. It has been evaluated in a phase I clinical study of hematologic malignancies. ${ }^{43}$

Reactivating apoptosis, the Bcl2 inhibitor venetoclax works independently from the presence of TP 53 mutations and has shown clinical activity in AML and MDS/AML. ${ }^{44,45}$ AML patients with epigenetic regulating and splicing gene mutations responded to venetoclax plus low-dose cytarabine in $68 \%$ of cases.

\section{Genetic Predisposition to MDS}

The WHO 2016 classification $^{1}$ introduced the category of myeloid neoplasms with familial predisposition (Figure 3). Awareness has been increasing of a possible inherited genetic defect in younger patients with MDS. In addition, the detection of such predisposing conditions has become easier and more frequent with the availability of deep sequencing techniques.

Although an inherited predisposition to cancer has long been known, the knowledge of a familial predisposition has been better defined for solid tumors with a subsequent effect on therapy and has been underestimated in MDS. Several genetic abnormalities and germline mutations have been correlated with a predisposition to MDS that could develop not only at young age but also in adult and elderly patients. The demonstration of SAMD9 and SAMD9L mutations in an adult with an alteration of chromosome 7 can lead to the diagnosis of MDS with an inherited genetic predisposition. Thus, recognition of such mutations and alterations is crucial for the choice of transplantation donors outside the family. Similarly, the cryptic variant of dyskeratosis congenita lacking the typical clinical signs can be diagnosed only using molecular techniques, provided that the evaluation of the mutations affecting the different
Figure 3 Germline Predisposition to Myeloid Neoplasia

\begin{tabular}{|c|c|}
\hline MUTATED GENES & DISEASE \\
\hline $\begin{array}{l}20 \text { different involved in DNA } \\
\text { repair,TP53 }\end{array}$ & Fanconi anemia, Li Fraumeni \\
\hline ELA2,HAX1, G6PC3, CSF3R,GFI1 & Severe congenital neutropenia \\
\hline 13 RPL, RPS genes, GATA1 & Diamond Blackfan Anemia \\
\hline $\begin{array}{l}\text { Different Telomere biology genes } \\
\text { TERC, TERT, DCK1 }\end{array}$ & $\begin{array}{l}\text { Dyskeratosis congenita, } \\
\text { Telomeropathies }\end{array}$ \\
\hline GATA2 & MDS/AML/CMML \\
\hline SAMD9, SAMD9L & $\begin{array}{l}\text { MDS, transient monosomy } 7 \text {, } \\
\text { MIRAGE syndrome }\end{array}$ \\
\hline ETV6 & Familial thrombocytopenia \\
\hline RUNX1 & Familial thrombocytopenia, AML \\
\hline ANKRD26 & $\begin{array}{l}\text { Familial thrombocytopenia, AML, } \\
\text { MDS, CML }\end{array}$ \\
\hline CEBPA & Familial AML \\
\hline DDX41 & Familial AML/MDS \\
\hline
\end{tabular}

Abbreviations: $\mathrm{AML}=$ acute myeloid leukemia; $\mathrm{CMML}=$ chronic myelomonocytic leukemia; MDS = myelodysplastic syndrome. Modified from Niemeyer CM oral communication.

telomere biology genes is included in the MDS routine diagnostic panel.

Lindsley et $\mathrm{al}^{32}$ identified a group of MDS patients aged $<40$ years with compound heterozygous mutations in the ShwachmanDiamond syndrome-associated SBDS gene. These patients had a poor prognosis, and all patients with biallelic SBDS mutations presented with somatic TP53 mutations. None had a recognized diagnosis of Shwachman-Diamond syndrome. ${ }^{32}$ Similarly, awareness in the nonpediatric setting of the clinical manifestations of congenital alterations that can mimic MDS (and, in fact, can predispose to MDS/AML, such as familial thrombocytopenia with germline ANKRD26 mutation) can avoid mistreatment of wrongly diagnosed MDS. ${ }^{46}$

Finally, a different situation is present in the case of inherited disease such as congenital neutropenia, in which clonal hematopoiesis and progression to MDS and AML is not determined by hematopoietic stresses such as therapy with granulocyte colonystimulating factors and the mutation burden is not increased. ${ }^{47}$

Patients with de novo MDS presenting at a younger age ( $<50$ years), patients with MDS and a family history of AML, and patients with MDS and peculiar extrahematologic symptoms should prompt molecular assessment for inherited genetic conditions predisposing to MDS. ${ }^{48}$ A series of actions should, therefore, be introduced into the routine examination of these peculiar cases. These include taking an accurate family and personal history to search for signs and symptoms of congenital syndromes, performing a mutational analysis for the genes involved in inherited predisposition, and selection of an accurate HSCT donor to completely avoid a related-matched donor generating slow engraftment and, frequently, donor-derived leukemia. The most relevant implication is the need for family genetic counseling, given the known anticipation of onset through the generations.

\section{Inflammation and MDS}

MDS research has recently focused on inflammation. The presence of inflammation increases with age ("inflammaging") and 
could be the trigger to clonal hematopoiesis. A chronic mild multifactorial inflammatory condition could induce an innate immune response and evolve into an oligoclonal $\mathrm{T}$-cell response, resulting in subversion of the immune response and progression from clonal hematopoiesis to low-risk MDS and then high-risk MDS (Kordasti, personal communication, 2018).

In contrast, the presence of CHIP has been shown to correlate with the development of coronary heart disease. ${ }^{49}$ Patients with CHIP had a 4 times greater risk of myocardial infarction, and 2 TET2 knockout mouse models had an increased atherogenesis and markers of activated inflammation. ${ }^{49}$ Finally, blocking inflammasome impairs the development of atherosclerosis.

\section{Methylation Abnormalities and Response to HMAs}

The HMAs azacitidine and decitabine are the standard treatment of MDS. Patients respond to these therapies in $40 \%$ to $60 \%$ of cases, transiently, and the dysplastic clone will be maintained despite hematologic improvement. The resistance to HMA treatment is, at present, one of the major problems in the management of MDS. Prediction of the response and distinction of the causes of primary versus secondary resistance are of paramount importance.

The aberrant methylation typical of MDS is determined by the presence of recurrent mutations in epigenetic regulatory genes, with a frequent DNA hypermethylation phenotype and decreased 5-hydroxymethylcytosine formation. Restoration of function of 1 of these genes, such as TET2, will eliminate progression of the disease. $^{50}$

The differences in the mode of action and activity between azacitidine and decitabine rely on different DNA uptake, with the azacitidine intracellular dynamic determinant for activity, but probably also on other factors. ${ }^{51}$ The mechanisms of resistance to HMAs are multifactorial. ${ }^{52}$ The recently demonstrated markers of sensitivity such as TET2 mutations, ${ }^{53}$ TP53 mutations, ${ }^{54}$ overexpression of nucleotide metabolizing enzymes, such as UCK1, ${ }^{55}$ and cytokines CXCL4 and CXCL7 ${ }^{56}$ must be consolidated. Novel schedules, doses, ${ }^{57}$ and combinations with numerous other agents have been investigated to increase the efficacy of these drugs and have been previously reported. ${ }^{58-60}$

New HMAs are under evaluation in the hopes of obtaining increased clinical efficacy. These include guadecitabine, previously known as SGI-110, a dinucleotide of decitabine and deoxyguanosine, that prolongs the in vivo exposure of decitabine by protecting it from deamination. ${ }^{61}$ An oral formulation of decitabine, ASTX727 (decitabine $35 \mathrm{mg} /$ cedazuridine $100 \mathrm{mg}$ ) is under evaluation in ongoing clinical trials of MDS. ${ }^{62}$

\section{Disclosure}

The authors have stated that they have no conflicts of interest.

\section{References}

1. Arber DA, Orazi A, Hasserjian R, et al. The 2016 revision to the World Health Organization classification of myeloid neoplasms and acute leukemia. Blood 2016; 127:2391-405.

2. Valent P, Orazi A, Steensma DP, et al. Proposed minimal diagnostic criteria for myelodysplastic syndromes (MDS) and potential pre-MDS conditions. Oncotarget 2017; 8:73483-500.
3. Westers TM, Ireland R, Kern W, et al. Standardization of flow cytometry in myelodysplastic syndromes: a report from an international consortium and the European LeukemiaNet Working Group. Leukemia 2012; 26:1730-41.

4. Schanz J, Tüchler H, Solé F, et al. New comprehensive cytogenetic scoring system for primary myelodysplastic syndromes (MDS) and oligoblastic acute myeloid leukemia after MDS derived from an international database merge. J Clin Oncol 2012; 30:820-9

5. Greenberg PL, Tuechler H, Schanz J. Revised international prognostic scoring system for myelodysplastic syndromes. Blood 2012; 120:2454-65.

6. Greenberg P, Cox C, LeBeau MM, et al. International scoring system for evaluating prognosis in myelodysplastic syndromes. Blood 1997; 89:2079-88.

7. Malcovati L, Della Porta MG, Strupp C, et al. Impact of the degree of anemia on the outcome of patients with myelodysplastic syndrome and its integration into the WHO classification-based prognostic scoring system (WPSS). Haematologica $2011 ; 96: 1433-40$

8. Bejar R. Myelodysplastic syndromes diagnosis: what is the role of molecular testing? Curr Hematol Malig Rep 2015; 10:282-91.

9. Papaemmanuil E, Gerstung M, Malcovati L, et al. Clinical and biological implications of driver mutations in myelodysplastic syndromes. Blood 2013; 122:3616-27.

10. Haferlach T, Nagata Y, Grossmann V, et al. Landscape of genetic lesions in 944 patients with myelodysplastic syndromes. Leukemia 2014; 28:24.

11. Kennedy JA, Ebert BL. Clinical implications of genetic mutations in myelodysplastic syndrome. J Clin Oncol 2017; 35:968-74.

12. Makishima H, Yoshizato T, Yoshida K, et al. Dynamics of clonal evolution in myelodysplastic syndromes. Nat Genet 2017; 49:204-12.

13. Chen GL, Prchal JT. X-linked clonality testing: interpretation and limitations. Blood 2007; 110:1411-9.

14. Genovese G, Kähler AK, Handsaker RE, et al. Clonal hematopoiesis and bloodcancer risk inferred from blood DNA sequence. $N$ Engl J Med 2014; 371:2477-87.

15. Jaiswal S, Fontanillas P, Flannick J, et al. Age-related clonal hematopoiesis associated with adverse outcomes. N Engl J Med 2014; 371:2488-98.

16. Xie M, Lu C, Wang J, et al. Age-related mutations associated with clonal hematopoietic expansion and malignancies. Nat Med 2014; 20:1472-8.

17. Steensma DP, Bejar R, Jaiswal S, et al. Clonal hematopoiesis of indeterminate potential and its distinction from myelodysplastic syndromes. Blood 2015; 126: $9-16$.

18. Bejar R. CHIP, ICUS, CCUS and other four-letter words. Leukemia 2017; 31: 1869-71.

19. Gillis NK, Ball M, Zhang Q, et al. Clonal haemopoiesis and therapy-related myeloid malignancies in elderly patients: a proof-of-concept, case-control study. Lancet Oncol 2017; 18:112-21.

20. Coombs CC, Zehir A, Devlin SM, et al. Therapy-related clonal hematopoiesis in patients with non-hematologic cancers is common and associated with adverse clinical outcomes. Cell Stem Cell 2017; 21:374-82.e4.

21. Malcovati L, Gallì A, Travaglino E, et al. Clinical significance of somatic mutation in unexplained blood cytopenia. Blood 2017; 129:3371-8.

22. Kosmider O, Passet M, Santini V, et al. Are somatic mutations predictive of response to erythropoiesis stimulating agents in lower risk myelodysplastic syndromes? Haematologica 2016; 101:e280-3.

23. Montalban-Bravo G, Takahashi K, Patel K, et al. Impact of the number of mutations in survival and response outcomes to hypomethylating agents in patients with myelodysplastic syndromes or myelodysplastic/myeloproliferative neoplasms. Oncotarget 2018; 9:9714-27.

24. Santini V, Fenaux P, Giagounidis A, et al. Impact of somatic gene mutations on response to lenalidomide (LEN) in IPSS lower-risk myelodysplastic syndromes (MDS) patients (Pts) without $\operatorname{Del}(5 \mathrm{q})$ and ineligible for or refractory to erythropoiesis-stimulating agents (ESAs). Blood 2016; 128:225.

25. Bejar R, Stevenson KE, Caughey B, et al. Somatic mutations predict poor outcome in patients with myelodysplastic syndrome after hematopoietic stem-cell transplantation. J Clin Oncol 2014; 32:2691-8.

26. Meggendorfer M, Haferlach C, Kern W, et al. Molecular analysis of myelodysplastic syndrome with isolated deletion of the long arm of chromosome 5 reveals a specific spectrum of molecular mutations with prognostic impact: a study on 123 patients and 27 genes. Haematologica 2017; 102:1502-10.

27. Scharenberg C, Giai V, Pellagatti A, et al. Progression in patients with low- and intermediate-1-risk del(5q) myelodysplastic syndromes is predicted by a limited subset of mutations. Haematologica 2017; 102:498-508.

28. Malcovati L, Papaemmanuil E, Ambaglio I, et al. Driver somatic mutations identify distinct disease entities within myeloid neoplasms with myelodysplasia. Blood 2014; 124:1513-21.

29. Malcovati L, Karimi M, Papaemmanuil E, et al. SF3B1 mutation identifies a distinct subset of myelodysplastic syndrome with ring sideroblasts. Blood 2015; 126:233-41.

30. Sallman DA, Tanaka TN, List A, Bejar R. SOHO state of the art update and next questions: biology and treatment of myelodysplastic syndromes. Clin Lymphome Myeloma Leuk 2017; 17:613-20.

31. Della Porta MG, Gallì A, Bacigalupo A, et al. Clinical effects of driver somatic mutations on the outcomes of patients with myelodysplastic syndromes treated with allogeneic hematopoietic stem-cell transplantation. J Clin Oncol 2016; 34: 3627.

32. Lindsley RC, Saber W, Mar BG, et al. Prognostic mutations in myelodysplastic syndrome after stem-cell transplantation. N Engl J Med 2017; 376:536-47.

33. Yoshizato T, Nannya Y, Atsuta Y, et al. Genetic abnormalities in myelodysplasia and secondary acute myeloid leukemia: impact on outcome of stem cell transplantation. Blood 2017; 129:2347-58. 


\section{SOHO Updates and Open Questions for MDS}

34. Maciejewski JP, Balasubramanian SK. Clinical implications of somatic mutations in aplastic anemia and myelodysplastic syndrome in genomic age. Hematol Am Soc Hematol Educ Program 2017; 2017:66-72.

35. DiNardo CD, De Botton S, Stein EM, et al. Ivosidenib (AG-120) in mutant IDH1 AML and advanced hematologic malignancies: results of a phase 1 dose escalation and expansion study. Blood 2017; 130:725.

36. Stein EM, DiNardo CD, Pollyea DA, et al. Enasidenib in mutant IDH2 relapsed or refractory acute myeloid leukemia. Blood 2017; 130:722-31.

37. Hong DS, Kurzrock R, Naing A, et al. A phase I, open-label, single-arm, doseescalation study of E7107, a precursor messenger ribonucleic acid (pre-mRNA) splicesome inhibitor administered intravenously on days 1 and 8 every 21 days to patients with solid tumors. Invest New Drugs 2014; 32:436-44.

38. Buonamici S, Yoshimi A, Thomas M, et al. H3B-8800, an orally bioavailable modulator of the SF3b complex, shows efficacy in spliceosome-mutant myeloid malignancies. Blood 2016; 128:966.

39. Platzbecker U, Germing U, Götze KS, et al. Luspatercept for the treatment of anaemia in patients with lower-risk myelodysplastic syndromes (PACE-MDS): a multicentre, open-label phase 2 dose-finding study with long-term extension study. Lancet Oncol 2017; 18:1338-47.

40. Komrokji R, Garcia-Manero G, Ades L, et al. Sotatercept with long-term extension for the treatment of anaemia in patients with lower-risk myelodysplastic syndromes: a phase 2, dose-ranging trial. Lancet Haematol 2018; 5:e63-72.

41. Reis B, Jukofsky L, Chen G, et al. Acute myeloid leukemia patients' clinical response to idasanutlin (RG7388) is associated with pre-treatment MDM2 protein expression in leukemic blasts. Haematologica 2016; 101:e185-8.

42. Zhang Q, Bykov VJN, Wiman KG, et al. APR-246 reactivates mutant p53 by targeting cysteines 124 and 277. Cell Death Dis 2018; 9:439.

43. Deneberg S, Cherif H, Lazarevic V, et al. An open-label phase I dose-finding study of APR-246 in hematological malignancies. Blood Cancer I 2016; 6:e447.

44. DiNardo CD, Rausch CR, Benton C, et al. Clinical experience with the BCL2inhibitor venetoclax in combination therapy for relapsed and refractory acute myeloid leukemia and related myeloid malignancies. Am J Hematol 2018; 93:401-7.

45. DiNardo CD, Pratz KW, Letai A, et al. Safety and preliminary efficacy of venetoclax with decitabine or azacitidine in elderly patients with previously untreated acute myeloid leukaemia: a non-randomised, open-label, phase $1 \mathrm{~b}$ study. Lancet Oncol 2018; 19:216-28.

46. Zaninetti C, Santini V, Tiniakou M, et al. Inherited thrombocytopenia caused by ANKRD26 mutations misdiagnosed and treated as myelodysplastic syndrome: report on two cases. I Thromb Haemost 2017; 15:2388-92.

47. Xia J, Miller CA, Baty J, et al. Somatic mutations and clonal hematopoiesis in congenital neutropenia. Blood 2018; 131:408-16.

48. Tawana K, Drazer MW, Churpek JE. Universal genetic testing for inherited susceptibility in children and adults with myelodysplastic syndrome and acute myeloid leukemia: are we there yet? Leukemia 2018. https://doi.org/10.1038/ s41375-018-0051-y. [Epub ahead of print].

49. Jaiswal S, Natarajan P, Silver AJ, et al. Clonal hematopoiesis and risk of atherosclerotic cardiovascular disease. N Engl J Med 2017; 377:111-21.

50. Cimmino L, Dolgalev I, Wang Y, et al. Restoration of TET2 function blocks aberrant self-renewal and leukemia progression. Cell 2017; 170:1079-95.e20.

51. Unnikrishnan A, Vo ANQ, Pickford R, et al. AZA-MS: a novel multiparameter mass spectrometry method to determine the intracellular dynamics of azacitidine therapy in vivo. Leukemia 2018; 32:900-10.

52. Unnikrishnan A, Papaemmanuil E, Beck D, et al. Integrative genomics identifies the molecular basis of resistance to azacitidine therapy in myelodysplastic syndromes. Cell Rep 2017; 20:572-85.

53. Bejar R, Lord A, Stevenson K, Bar-Natan M, Pérez-Ladaga A, Zaneveld J. TET2 mutations predict response to hypomethylating agents in myelodysplastic syndrome patients. Blood 2014; 124:2705-12.

54. Welch JS, Petti AA, Miller CA, et al. p53 and decitabine in acute myeloid leukemia and myelodysplastic syndromes. N Engl J Med 2016; 375:2023-36.

55. Valencia A, Masala E, Rossi A, et al. Expression of nucleoside-metabolizing enzymes in myelodysplastic syndromes and modulation of response to azacitidine. Leukemia 2014; 28:621-8

56. Meldi K, Qin T, Buchi F, et al. Specific molecular signatures predict decitabine response in chronic myelomonocytic leukemia. J Clin Invest 2015; 125: 1857-72.

57. Short NJ, Garcia-Manero G, Montalban Bravo G. Low-dose hypomethylating agents (HMAs) are effective in patients (Pts) with low- or intermediate-1-risk myelodysplastic syndrome (MDS): a report on behalf of the MDS Clinical Research Consortium. Blood 2015; 126:94.

58. Shallis RM, Zeidan AM. More is less, less is more, or does it really matter? The curious case of impact of azacitidine administration schedules on outcomes in patients with myelodysplastic syndromes. BMC Hematol 2018; 18:4.

59. Ornstein MC, Mukherjee S, Sekeres MA. More is better: combination therapies for myelodysplastic syndromes. Best Pract Res Clin Haematol 2015; 28 : 22-31.

60. Daver N, Boddu P, Garcia-Manero G, et al. Hypomethylating agents in combination with immune checkpoint inhibitors in acute myeloid leukemia and myelodysplastic syndromes. Leukemia 2018; 32:1094-105.

61. Sebert M, Bally C, Peterlin P, et al. Results of a phase II study of guadecitabine (SGI-110) in higher risk MDS, CMML or low blast count AML patients refractory to or relapsing after azacitidine (AZA) treatment. Blood 2016; 128:347.

62. Garcia Manero G, Griffith E, Roboz GJ, et al. A phase 2 dose-confirmation study of oral ASTX727, a combination of oral decitabine with a cytidine deaminase inhibitor (CDAi) cedazuridine (E7727), in subjects with myelodysplastic syndromes (MDS). Blood 2017; 130:4274. 